\title{
THE RING OF POLYNOMIALS OVER A VON NEUMANN REGULAR RING
}

\author{
P. J. McCARTHY ${ }^{1}$
}

Abstract. It is shown that the ring of polynomials in one indeterminate over a commutative von Neumann regular ring with identity element is semihereditary.

Let $R$ be a commutative von Neumann regular ring with identity element. Soublin stated without proof in [4], and again in [5], that the polynomial ring $R[X]$ is coherent. Recently, Sabbagh proved that a ring of polynomials in any number of indeterminates over $R$ is coherent [3]. Carson obtained similar results for certain noncommutative rings [1]. In this note we prove the following result for the case of a single indeterminate.

\section{THEOREM. $\quad R[X]$ is semihereditary.}

By a result of Vasconcelos [6, Theorem 4.2] the assertion follows if we show that w.gl.dim $R[X] \leqq 1$ and that the annihilator of each element of $R[X]$ is finitely generated.

Since w.gl.dim $R=0$, it is a consequence of a result of Jensen [2, Theorem 2] that w.gl.dim $R[X]=1$.

Let $f(X)=a_{0}+a_{1} X+\cdots+a_{n} X^{n} \in R[X]$ and let $A$ be the annihilator of $f(X)$ in $R[X]$. For $i=0, \cdots, n$, let $e_{i}$ be an idempotent element in $R$ such that $R a_{i}=R e_{i}$. Let $e=\left(1-e_{0}\right) \cdots\left(1-e_{n}\right)$. Clearly, $R[X] e \subseteq A$. Let $g(X)=b_{0}+b_{1} X+\cdots+b_{k} X^{k} \in A$ : we show by double induction on $k$ and $n$ that $g(X) \in R[X] e$. Thus, $A=R[X] e$.

If $k=0$, then $f(X) b_{0}=0$ and so $e_{i} b_{0}=0$ for $i=0, \cdots, n$. Hence $b_{0}=b_{0} e \in R[X] e$

Suppose $k>0$ and that, for arbitrary $n$, if $h(X) \in A$ and $\operatorname{deg} h(X)<k$, then $h(X) \in R[X] e$. If $n=0$, then $e_{0} g(X)=0$ and $g(X)=g(X) e \in R[X] e$. Suppose $n>0$ and that the inclusion we are asserting holds when $f(X)$ is replaced by a polynomial of degree less than $n$.

Received by the editors October 5, 1972.

AMS (MOS) subject classifications (1970). Primary 16A30, 13F20; Secondary 13A15.

Key words and phrases. Commutative von Neumann regular ring, polynomial ring, coherent ring, semihereditary ring.

${ }^{1}$ Research supported by University of Kansas General Research Fund Grant 3919-5038.

(c) American Mathematical Society 1973 
Since $e_{n} b_{k}=0$ we have $\operatorname{deg} e_{n} g(X)<k$, and certainly $f(X) e_{n} g(X)=0$. Hence, by the induction assumption on $k$, there is a polynomial $m(X) \in$ $R[X]$ such that $e_{n} g(X)=e m(X)$. Then $e_{n} g(X)=e_{n}^{2} g(X)=e_{n} e m(X)=0$, and since $a_{n}$ is a multiple of $e_{n}$ it follows that $a_{n} g(X)=0$. Consequently,

$$
\left(f(X)-a_{n} X^{n}\right) g(X)=0,
$$

and by the induction assumption on $n$,

$$
g(X) \in R[X]\left(1-e_{0}\right) \cdots\left(1-e_{n-1}\right) .
$$

Therefore, $g(X)=g(X)\left(1-e_{n}\right) \in R[X] e$.

\section{REFERENCES}

1. A. B. Carson, Coherence of polynomial rings over semisimple algebraic algebras, Proc. Amer. Math. Soc. 34 (1972), $20-24$.

2. C. U. Jensen, On homological dimensions of rings with countably generated ideals, Math. Scand. 18 (1966), 97-105. MR 34 \#7611.

3. G. Sabbagh, Coherence of polynomial rings and bounds in polynomial ideals, J. Algebra (to appear).

4. J.-P. Soublin, Anneaux cohérents, C. R. Acad. Sci. Paris Sér. A-B 267 (1968), A183-A186. MR 38 \#1121.

5. —_, Anneaux et modules cohérents, J. Algebra 15 (1970), 455-472. MR 41 \#5422.

6. W. V. Vasconcelos, On finitely generated flat modules, Trans. Amer. Math. Soc. 138 (1969), 505-512. MR 39 \#199.

Department of Mathematics, University of Kansas, LaWrence, Kansas 66044 